\title{
Exclusive glueball production in high-energy nucleus-nucleus collisions
}

\author{
M. V. T. Machado ${ }^{1}$ and M. L. L. da Silva ${ }^{2}$ \\ ${ }^{1}$ High Energy Physics Phenomenology Group, High Energy Particle Phenomenology Group - Instituto de Fisica - Universidade Federal do \\ Rio Grande do Sul, Caixa Postal 15051, CEP 91501-970, Porto Alegre, Rio Grande do Sul, Brazil \\ ${ }^{2}$ Centro de Ciências Fisicas e Matemáticas, Departamento de Fisica, Universidade Federal de Santa Catarina, Bairro Trindade. Caixa Postal \\ 476, CEP 88040-970, Florianopolis, Santa Catarina, Brazil
}

(Received 20 October 2010; revised manuscript received 26 November 2010; published 25 January 2011)

\begin{abstract}
The cross sections for the glueball candidates in quasireal photon-photon collisions and on central diffraction processes (i.e., double Pomeron exchange) in heavy-ion interactions at the Relativistic Heavy-Ion Collider (RHIC) and the Large Hadron Collider (LHC) are computed. The rates for these distinct production channels are compared, and they may be a fruitful approach to the investigation of glueballs.
\end{abstract}

DOI: 10.1103/PhysRevC.83.014907

PACS number(s): 25.75.Cj, 12.38.-t, 12.39.Mk, 14.40.-n

\section{INTRODUCTION}

The gluon self-coupling in QCD opens the possibility of existing bound states of pure gauge fields known as glueballs. Glueballs $(G)$ are predicted by several theoretical formalisms and by lattice calculations. For a comprehensive review of the current status of theoretical and experimental aspects of glueball studies, we refer readers to Refs. [1] and [2], respectively. Many mesons have been considered as good candidates for the lightest glueball in the spectrum, and in particular the scalar sector $\left(J^{P C}=0^{++}\right)$seems promising. The mesons $f_{0}(1500)$ and the $f_{0}(1710)$ have been considered the principal candidates for the scalar glueball $[3,4]$. However, in this mass region, the glueball state will mix strongly with nearby $q \bar{q}$ states [4,5]. More recently, the Beijing Spectrometer (BES) collaboration observed a new resonance called $X(1835)$ [6]. It is an important candidate for a glueball, and the nature of meson $X(1835)$ has several interpretations. Some consider it a pseudoscalar glueball $\left(J^{\mathrm{PC}}=0^{-+}\right)$as first suggested in Ref. [7] and afterward in Ref. [8].

Recently, the clean topologies of exclusive particle production in electromagnetic interactions such as hadron-hadron and nucleus-nucleus collisions mediated by colorless exchanges such the QCD Pomeron or two photons have attracted increasing interest [9]. The cross sections for these processes are smaller than the corresponding inclusive production channels, which is compensated by a more favorable signal/background relation. Experimentally, exclusive events are identified by large rapidity gaps on both sides of the produced central system and the survival of both initial-state particles scattered at very forward angles with respect to the beam.

Here, we focus on exclusive glueball production in two-photon and Pomeron-Pomeron interactions in coherent nucleus-nucleus collisions at high-energy colliders [the Relativisitic Heavy-Ion Collider (RHIC) and the Large Hadron Collider (LHC)]. In these cases, the photon flux scales as the square charge of the beam, $Z^{2}$, and then the corresponding cross section is highly enhanced by a factor $\propto Z^{4} \approx 10^{7}$ for gold or lead nuclei. A competing channel, which produces similar final-state configurations, is the central diffraction (CD) process. Such a reaction is modeled in general by the two-Pomeron interaction. Experimentally, the separation of these channels is somewhat difficult, and from atheoretical point of view the Pomeron-Pomeron interactions are subject to large uncertainties at collider energies. One goal of the present work is to compare the cross sections for these two channels in the production of glueball candidates. This paper is organized as follows: In the next section, we present the main expressions for cross-sectional calculation of two-photon and Pomeron-Pomeron processes, and in last section, we show and discuss the numerical results.

\section{CROSS-SECTIONAL CALCULATION}

Let us start with the glueball production in photonphoton scattering at coherent heavy-ion collisions using the Weizsäcker-Williams approximation Equivalent Photon Approximation (EPA). In such an approach, the cross section for a two-quasireal-photon process to produce a glueball state, $G$, at center-of-mass energy $W_{\gamma \gamma}$ factorizes into the product of the elementary cross section for $\gamma \gamma \rightarrow G$ convoluted with the equivalent photon spectra from the colliding ions [9]:

$\sigma_{\gamma \gamma}(A A \rightarrow A+G+A)=\int \frac{d k_{1}}{k_{1}} \frac{d k_{2}}{k_{2}} \frac{d n_{\gamma}}{d k_{1}} \frac{d n_{\gamma}}{d k_{2}} \sigma(\gamma \gamma \rightarrow G)$,

where $k_{1,2}$ are the photon energies and $d n / d k$ is the photon flux at the energy $k$ emitted by the hadron $A$. The photon energies determine the center-of-mass energy $W_{\gamma \gamma}=\sqrt{4 k_{1} k_{2}}$ and the rapidity $Y$ of the produced system. Namely, one has $k_{1,2}=\left(W_{\gamma \gamma} / 2\right) \exp ( \pm Y)$ and $Y=(1 / 2) \ln \left(k_{1} / k_{2}\right)$. In addition, $\sqrt{s_{N N}}$ is the center-of-mass energy of the ion-ion system and the Lorentz relativistic factor is given by $\gamma_{L}=$ $\sqrt{s_{N N}} /\left(2 m_{N}\right)$. In particular, in the numerical calculations we use $\sqrt{s_{N N}}=0.2(5.5) \mathrm{TeV}$ and $\gamma_{L}=109$ (2930) for RHIC (LHC).

In the EPA approximation, the flux of equivalent photons from a relativistic particle of charge $Z$ is determined from the Fourier transform of its electromagnetic field. For an extended charge with electromagnetic form factor $F_{A}\left(Q^{2}\right)$, the energy spectrum can be computed as

$$
\frac{d n_{\gamma / A}(x)}{d k}=\frac{\alpha Z^{2}}{\pi} \frac{A(x)}{x} \int \frac{Q^{2}-Q_{\min }^{2}}{Q^{4}}\left|F_{A}\left(Q^{2}\right)\right|^{2} d Q^{2},
$$

where $x=k / E$ is the fraction of the beam energy carried by the photon and $A(x)=1-x+\left(1 / 2 x^{2}\right)$. Moreover, $\alpha=$ 
$1 / 137$ and $Q^{2}$ is the four-momentum transfer squared from the charge, with $Q_{\min }^{2} \approx\left(x m_{N}\right)^{2} /(1-x)$.

The glueball production in two-photon fusion can be calculated using the narrow-resonance approximation [10]:

$$
\sigma(\gamma \gamma \rightarrow G)=(2 J+1) \frac{8 \pi^{2}}{M_{G}} \Gamma(G \rightarrow \gamma \gamma) \delta\left(W_{\gamma \gamma}^{2}-M_{G}^{2}\right) \text {, }
$$

where $\Gamma(G \rightarrow \gamma \gamma)$ is the partial two-photon decay width of $G, M_{G}$ is the glueball mass, and $J$ is the spin of the state $G$. Here, we compute the production rates for the mesons $f_{0}(1500), f_{0}(1710)$, and $X(1835)$ [11], respectively, because they have been mentioned as possible glueball candidates by phenomenologists [1,2].

Some important comments are in order. The predictions for the two-photon component are in practice somewhat difficult as the branching ratios have not been measured. To compute numerical values for the meson (glueball) cross section in twophoton reactions, estimates for the two-photon decay widths are needed. The determination of them depend upon whether the meson state is a pure quarkonium, pure gluonic, or a mixed hybrid state. For a pure quarkonium state, the width can be related (at leading order) to the two-gluon width, $\Gamma(q \bar{q} \rightarrow g g)$. Namely, $\Gamma(q \bar{q} \rightarrow \gamma \gamma) \simeq D_{c} e_{q}^{4}\left(\alpha / \alpha_{s}\right)^{2} \Gamma(q \bar{q} \rightarrow g g)$, where $D_{c}=9 / 2$ is the color factor and $e_{q}$ is the relevant quark charge. One can estimate the two-gluon width from the total width for the meson state and the theoretical expectation that the $q \bar{q} \rightarrow g g$ branching ratio ${ }^{1}$ is of order $\alpha_{s}^{2}$ [12]. In the case of a pure gluonic state, the two-photon width can be computed using a nonrelativistic gluon bound-state model as performed, for instance, in Ref. [13]. There, the unknown parameters, the digluon wave function or its first/second derivative at the origin, are determined by using measured values of $\Gamma(J / \psi \rightarrow G \gamma)$.

Now, we compute estimates for the two-photon widths assuming pure $q \bar{q}$ and pure gluonic resonances, respectively. For the first case, as discussed previously, we take $\Gamma(R \rightarrow \gamma \gamma)=$ $e_{q}^{4}(3 \alpha)^{2} \Gamma_{\text {tot }}(R) / 2$. Using the Particle Data Group (PDG) average values for the total width, one gets $\Gamma\left[f_{0}(1500) \rightarrow \gamma \gamma\right] \simeq$ $0.3 \mathrm{keV}, \Gamma\left[f_{0}(1710) \rightarrow \gamma \gamma\right] \simeq 0.4 \mathrm{keV}$, and $\Gamma[X(1835) \rightarrow$ $\gamma \gamma] \simeq 0.2 \mathrm{keV}$. The corresponding cross sections using these theoretical estimates for the width are 3 (158) $\mu \mathrm{b}, 3.4$ (216) $\mu \mathrm{b}$, and 1.1 (84) $\mu \mathrm{b}$ at RHIC (LHC). If we are conservative, one can consider the experimental upper bounds for the two-photon widths as $f_{0}(1500)$ and $f_{0}(1710)$. This procedure gives an upper limit of the cross section for those resonances in peripheral collisions. The ALEPH experiment [3] studied the $\gamma \gamma$ production of those glueball candidates via their decay to $\pi^{+} \pi^{-}$and the following limits ${ }^{2}$ were determined: $\Gamma\left[f_{0}(1500) \rightarrow \gamma \gamma\right] \leqslant 1.08 \mathrm{keV}$ and $\Gamma\left[f_{0}(1710) \rightarrow \gamma \gamma\right] \leqslant$

\footnotetext{
${ }^{1}$ For pure glueball resonance, $G$, the branching ratio is $B(G \rightarrow$ $g g) \simeq 1$, whereas mixing states will give intermediate values of branching ratio.

${ }^{2}$ Here, we consider the ALEPH limits $\Gamma\left[\gamma \gamma \rightarrow f_{0}(1500)\right]$. $B\left[f_{0}(1500) \rightarrow \pi^{+} \pi^{-}\right]<0.31 \quad \mathrm{keV}, \quad \Gamma\left[\gamma \gamma \rightarrow f_{0}(1710)\right]$. $B\left[f_{0}(1710) \rightarrow \pi^{+} \pi^{-}\right]<0.55 \mathrm{keV}$ and taking the branching ratios $0.30 \pm 0.07$ and $0.026 \pm 0.016$ [3], respectively.
}

$21.25 \mathrm{keV}$. Using those limits, the corresponding cross sections are of order $0.95 \mathrm{mb}(20 \mu \mathrm{b})$ for $f_{0}(1500)$ and $11.5 \mathrm{mb}$ $(180 \mu \mathrm{b})$ for $f_{0}(1710)$ at LHC (RHIC) energies. We quote Ref. [14] for a comparison of our results with a wide class of theoretical models and exotic QCD states in the meson production in photon-photon process.

For a pure glueball resonance, we follow [13], adapted for the candidates considered here. Namely, assuming the $f_{0}$ resonances as states with $J=L=0$, then Eq. (54) of Ref. [13] has been used, where we take the PDG values for the radiative $J / \psi$ decays in the following channels: $\psi \rightarrow \gamma f_{0}(1500) \rightarrow$ $\gamma \pi \pi$ and $\psi \rightarrow \gamma f_{0}(1710) \rightarrow \gamma K \bar{K}$. Assuming the $X(1835)$ resonance to be a state with $J=0$ and $L=S=1$, we rely on Eq. (35) of [13] and use the PDG value for the decay channel $\psi \rightarrow \gamma X(1835) \rightarrow \gamma \pi^{+} \pi^{-} \eta^{\prime}$. Putting it all together, we estimate that the two-photon widths for a pure glueball resonance are $\Gamma\left[f_{0}(1500) \rightarrow \gamma \gamma\right] \simeq 0.77 \mathrm{eV}, \Gamma\left[f_{0}(1710) \rightarrow\right.$ $\gamma \gamma] \simeq 7.03 \mathrm{eV}$, and $\Gamma[X(1835) \rightarrow \gamma \gamma] \simeq 0.021 \mathrm{keV}$. Notice that for the $X(1835)$ a larger width is predicted [8], being of order $1.1 \mathrm{keV}$. The widths are about three orders of magnitude smaller than for pure $q \bar{q}$ states. Therefore, as the two-photon cross section scales as $(2 J+1) \Gamma(R \rightarrow \gamma \gamma)$, Eq. (3), one can consider the experimental feasibility of using peripheral heavy-ion collisions to determine the nature of the resonances discussed previously. The values for the corresponding widths and corresponding cross sections estimates are shown in Table I.

Now, we address the Pomeron-Pomeron channel. In particular, we focus on the central diffraction (double Pomeron exchange, DPE) in nucleus-nucleus interactions. As a starting point, we compute the DPE proton-proton cross section, making use of the Bialas-Landshoff $[15,16]$ approach. We believe that this nonperturbative approach is a reasonable choice due to the light mass of glueball candidates considered in the present calculation. For a perturbative QCD guided calculation, we quote the recent work in Ref. [17], where the exclusive scalar $f_{0}(1500)$ meson production is carefully investigated. Here, we are particularly interested in the exclusive and central inclusive (central inelastic) DPE production of glueball states. In the exclusive DPE event, the central object $G$ is produced alone, separated from the outgoing hadrons by rapidity gaps, $p p \rightarrow p+$ gap $+G+$ gap $+p$. In the central inclusive DPE event, an additional radiation accompanying the central object is allowed. In the approach we use, Pomeron exchange corresponds to the exchange of a pair of nonperturbative gluons that takes place between a pair of colliding quarks. For DPE central inclusive $G$ production, we can neglect the additional gap-spoiling effect, the so-called Sudakov effect.

TABLE I. Cross sections for pure glueball candidate production through photon-photon fusion in electromagnetic nucleus-nucleus collisions at RHIC and LHC energies.

\begin{tabular}{lccc}
\hline \hline Glueball candidate & $\Gamma_{\gamma \gamma}(\mathrm{eV})$ & RHIC $(\mathrm{nb})$ & $\mathrm{LHC}(\mu \mathrm{b})$ \\
\hline$f_{0}(1500)$ & 0.77 & $14-9.3$ & $0.7-1.3$ \\
$f_{0}(1710)$ & 7.03 & $60-43$ & $3.8-8.6$ \\
$X(1835)$ & 0.021 & $0.11-0.09$ & $0.01-0.02$ \\
\hline \hline
\end{tabular}


The scattering matrix is given by

$$
\begin{aligned}
\mathcal{M}= & \mathcal{M}_{0}\left(\frac{s}{s_{1}}\right)^{\alpha\left(t_{2}\right)-1}\left(\frac{s}{s_{2}}\right)^{\alpha\left(t_{1}\right)-1} F\left(t_{1}\right) F\left(t_{2}\right) \\
& \times \exp \left[\beta\left(t_{1}+t_{2}\right)\right] S_{\text {gap }}(\sqrt{s}) .
\end{aligned}
$$

Here $\mathcal{M}_{0}$ is the amplitude in the forward scattering limit $\left(t_{1}=t_{2}=0\right)$. The standard Pomeron Regge trajectory is given by $\alpha(t)=1+\epsilon+\alpha^{\prime} t$ with $\epsilon \approx 0.08, \alpha^{\prime}=0.25 \mathrm{GeV}^{-2}$. The momenta of incoming (outgoing) protons are labeled by $p_{1}$ and $p_{2}\left(k_{1}\right.$ and $\left.k_{2}\right)$, whereas the glueball momentum is denoted by $P$. Thus, we can define the following quantities appearing in Eq. (4): $s=\left(p_{1}+p_{2}\right)^{2}, s_{1}=\left(k_{1}+P\right)^{2}, s_{2}=\left(k_{2}+P\right)^{2}, t_{1}=$ $\left(p_{1}-k_{1}\right)^{2}$, and $t_{2}=\left(p_{2}-k_{2}\right)^{2}$. The nucleon form factor is given by $F_{p}(t)=\exp (b t)$ with $b=2 \mathrm{GeV}^{-2}$. The phenomenological factor $\exp \left[\beta\left(t_{1}+t_{2}\right)\right]$ with $\beta=1 \mathrm{GeV}^{-2}$ takes into account the effect of the momentum transfer dependence of the nonperturbative gluon propagator. The factor $S_{\text {gap }}$ takes the gap survival effect into account, that is, the probability $\left(S_{\text {gap }}^{2}\right)$ of the gaps not being populated by secondaries produced in the soft rescattering. For our purpose here, we consider $S_{\text {gap }}^{2}=0.032$ at $\sqrt{s}=5.5 \mathrm{TeV}$ in nucleon-nucleon collisions ${ }^{3}$ and $S_{\text {gap }}^{2}=0.15$ at $\sqrt{s}=200 \mathrm{GeV}$ (RHIC). In particular, for RHIC we have used an estimation using a simple one-channel eikonal model for the survival probability [18], whereas for the LHC energy we follow Ref. [19], which considers a two-channel eikonal model that embodies pion-loop insertions in the pomeron trajectory, diffractive dissociation, and rescattering effects. We refer to Ref. [20] for a detailed comparison between the two approaches and further discussions on model dependence of inputs and consideration of multichannel calculations.

Following the calculation presented in Ref. [16], we find $\mathcal{M}_{0}$ for colliding hadrons,

$$
\mathcal{M}_{0}=32 \alpha_{0}^{2} D_{0}^{3} \int d^{2} \vec{\kappa} p_{1}^{\lambda} V_{\lambda \nu}^{J} p_{2}^{\nu} \exp \left(-3 \vec{\kappa}^{2} / \tau^{2}\right),
$$

where $\kappa$ is the transverse momentum carried by each of the three gluons. $V_{\lambda \nu}^{J}$ is the $g g \rightarrow G^{J}$ vertex depending on the polarization $J$ of the $G^{J}$ glueball meson state. For the cases considered here, $J=0$, one obtains the following result [16,21]:

$$
p_{1}^{\lambda} V_{\lambda \nu}^{0} p_{2}^{\nu}=\frac{s \vec{\kappa}^{2}}{2 M_{G^{0}}^{2}} A
$$

where $A$ is expressed by the mass $M_{G}$ and the width $\Gamma(g g \rightarrow$ $G$ ) of the glueball meson through the relation

$$
A^{2}=8 \pi M_{G} \Gamma(g g \rightarrow G) \text {. }
$$

For obtaining the two-gluon decays widths, the following relation is used: $\Gamma(G \rightarrow g g)=B(G \rightarrow g g) \Gamma_{\text {tot }}(G)$. At this point, some discussion is in order. The two-gluon width depends on the branching fraction of the resonance $R$ to gluons,

\footnotetext{
${ }^{3}$ It is obtained using a parametric interpolation formula for the Khoze-Martin-Ryskin (KMR) survival probability factor [19] in the form $S_{\text {gap }}^{2}=a /\left[b+\ln \left(\sqrt{s / s_{0}}\right)\right]$ with $a=0.126, b=-4.988$, and $s_{0}=1 \mathrm{GeV}^{2}$. This formula interpolates between $\mathrm{CD}$ survival probabilities of $4.5 \%$ at Tevatron and $2.6 \%$ at the LHC.
}

$B(G \rightarrow g g)$, and its knowledge would give quantitative information on the glueball content of a particular resonance. As discussed before, it is a theoretical expectation [12] that $B[R(q \bar{q}) \rightarrow g g]=\mathcal{O}\left(\alpha_{s}^{2}\right) \simeq 0.1-0.2$, whereas $B[R(G) \rightarrow$ $g g] \simeq 1$. Here, we are conservative and assume the resonance to be a pure glueball. This fact translates into an upper bound for the exclusive DPE production as the cross section scales with $\Gamma(R \rightarrow g g)$. Following Ref. [22], the two-gluon width can be computed from the resonance branching fraction in $J / \psi$ radiative decay, $B(\psi \rightarrow \gamma G)$. For the candidates of interest here, one obtains

$$
\begin{aligned}
& B\left[G\left(0^{++}\right) \rightarrow g g\right]=\frac{8 \pi\left(\pi^{2}-9\right) B\left[\psi \rightarrow \gamma G\left(0^{++}\right)\right]}{c_{R} x\left|H_{J}(x)\right|^{2} \Gamma_{\text {tot }}} \frac{M_{\psi}^{2}}{M_{G}}, \\
& B\left[G\left(0^{-+}\right) \rightarrow g g\right]=\frac{8 \pi\left(\pi^{2}-9\right) B\left[\psi \rightarrow \gamma G\left(0^{-+}\right)\right]}{c_{R} x\left|H_{J}(x)\right|^{2} \Gamma_{\text {tot }}} \frac{M_{\psi}^{2}}{M_{G}},
\end{aligned}
$$

where the function $H_{J}(x)$ is determined in the nonrelativistic quark model (NRQM) (see appendix of Ref. [22]) and $c_{R}$ is a numerical constant $\left(C_{R}=1,2 / 3,5 / 2\right.$ for $J^{\mathrm{PC}}=$ $0^{-+}, 0^{++}, 2^{++}$, respectively). The masses of $J / \psi$ and of resonance are $M_{\psi}$ and $M_{G}$, respectively, and $x=1-\left(M_{G}^{2} / M_{\psi}^{2}\right)$. Based on these equations, in Ref. [22] the following values for the branching fractions for scalar glueballs candidates are obtained: $B\left[f_{0}(1500)\right]=0.64 \pm 0.11, B\left[f_{0}(1710)\right]=0.52 \pm$ 0.07 . For the pseudoscalar $X$ the situation is less clear due to little information on its decaying channels in radiative $J / \psi$ decays. The authors in [22] have a prediction for $\eta$ resonance which gives $B[\eta(1410)]=0.9 \pm 0.2$. As the branching fraction scales as $1 / M_{G}$ in this theoretical model, an educated guess for the $X$ branching fraction would be $B[X(1835)]=\left(M_{\eta} / M_{X}\right) \cdot B[\eta(1410)]=0.69 \pm 0.15$. In the numerical calculations, we set the limit case $B[X(1835)]=1$ and notice that the branching would be about $30 \%$ smaller. The values for $\Gamma_{g g}$ used in our calculations are summarized in Table II. A consequence of the small deviation for the branching fraction in pure $q \bar{q}$ and glueball resonance is the difficulty in testing their nature using the exclusive diffractive data. An option would be to obtain, for instance, the differential cross section on angular distributions and then compare the predictions for each composition (pure $q \bar{q}$, mixing state, and pure glueball).

In addition, we use the parameters $\tau=1 \mathrm{GeV}$ and $D_{0} G^{2} \tau=30 \mathrm{GeV}^{-1}$ [16], where $G$ is the scale of the process-independent nonperturbative quark gluon coupling. An indirect determination of unknown parameter $\alpha_{0}=G^{2} / 4 \pi$

TABLE II. Cross sections for inclusive (inc.) and exclusive (exc.) glueball production in the DPE process for RHIC and LHC energies.

\begin{tabular}{lccc}
\hline \hline Glueball & $\Gamma_{g g}(\mathrm{MeV})$ & RHIC $(\mathrm{mb})$ & $\mathrm{LHC}(\mathrm{mb})$ \\
\hline$f_{0}(1500)$ & \multirow{2}{*}{6.8} & $0.63 \pm 0.21$ (inc.) & $0.77 \pm 0.51$ (inc.) \\
& & $0.40 \pm 0.14$ (exc.) & $0.50 \pm 0.32$ (exc.) \\
$f_{0}(1710)$ & 70.2 & $0.68 \pm 0.26$ (inc.) & $0.80 \pm 0.52$ (inc.) \\
& & $0.41 \pm 0.16$ (exc.) & $0.49 \pm 0.31$ (exc.) \\
$X(1835)$ & 70.27 & $0.64 \pm 0.24$ (inc.) & $0.77 \pm 0.50$ (inc.) \\
& & $0.38 \pm 0.14$ (exc.) & $0.45 \pm 0.29$ (exc.) \\
\hline \hline
\end{tabular}


has been found in Ref. [23] using experimental data for central inclusive dijet production cross section at Tevatron. Namely, the constraint $S_{\text {gap }}^{2}(\sqrt{s}=2 \mathrm{TeV}) / \alpha_{0}^{2}=0.6$, where $S_{\text {gap }}^{2}$ is the gap survival probability factor (absorption factor), has been found. Considering the KMR [19] value $S_{\text {gap }}^{2}=0.045$ for CD processes at Tevatron energy, one obtains $\alpha_{0}=0.274$.

The calculation presented here concerns the central inclusive process, where the QCD radiation accompanying the produced object is allowed. Therefore, in order to describe the exclusive processes where the central object is produced alone, we include the Sudakov survival factor $T(\kappa, \mu)$ [24] inside the loop integral over $\vec{\kappa}$. The Sudakov factor $T(\kappa, \mu)$ is the survival probability that a gluon with transverse momentum $\kappa$ remains untouched in the evolution up to the hard scale $\mu=M_{G} / 2$. The function $T(\kappa, \mu)$ is given by [24]

$$
\begin{aligned}
T(\kappa, \mu)= & \exp \left(-\int_{\vec{\kappa}^{2}}^{\mu^{2}} \frac{\alpha_{s}\left(\vec{k}^{2}\right)}{2 \pi} \frac{d \vec{k}^{2}}{\vec{k}^{2}}\right. \\
& \left.\times \int_{0}^{1-\delta}\left[z P_{g g}(z)+\sum_{q} P_{q g}(z)\right] d z\right),
\end{aligned}
$$

where $\delta=|\vec{k}| /(\mu+|\vec{k}|), P_{g g}(z)$ and $P_{q g}(z)$ are the DokshitzerGribov-Lipatov-Altarelli-Parisi spitting functions. In next section, we discuss the effect of introducing the Sudakov factor in the estimation of exclusive production in the PomeronPomeron channel.

In order to calculate the $A A$ cross section, the procedure presented in Ref. [25] is considered, where the central diffraction and single diffraction cross sections in nucleus-nucleus collisions are computed using the so-called criterion $C$ (we refer to Ref. [25] for further details). Using the profile function for two colliding nuclei, $T_{A B}=\int d^{2} \bar{b} T_{A}(\bar{b}) T_{B}(b-\bar{b})$, the final expression for $\mathrm{CD}$ cross section in $A A$ collisions is given by [25]

$$
\sigma_{A A}^{\mathrm{CD}}=A^{2} \int d^{2} b T_{A A}(b) \exp \left[-A^{2} \sigma_{p p}^{i n} T_{A A}(b)\right] \sigma_{p p}^{\mathrm{CD}},
$$

where $\sigma_{p p}^{\text {in }}$ and $\sigma_{p p}^{\mathrm{CD}}=S_{\text {gap }}^{2} \times \sigma_{p p}^{\mathrm{CD}}(\sqrt{s})$ are the inelastic and $\mathrm{CD}$ cross sections, respectively, in the proton-proton case.

Using Woods-Saxon nuclear densities and considering the inelastic cross section $\sigma_{p p}^{\text {in }}=73$ (49) $\mathrm{mb}$ for LHC (RHIC) energy, $\sqrt{s_{A A}}=5.5(0.2) \mathrm{TeV}$, we compute the CD cross section for nuclear collisions. The values for the inelastic cross section are obtained from DPMJET [26], where the scattering amplitude is parameterized using $\sigma_{\text {tot }}, \rho$ and elastic slope (these parameters are taken as fitted by the PHOJET model [27]). We notice that for LHC energy the effective atomic number dependence is proportional to $A^{1 / 3}$, which means that the nuclear CD cross section is only one order of magnitude larger than the nucleon-nucleon cross section. For completeness, we give the values of the DPE cross sections for the proton-proton case used in Eq. (9): $\sigma_{p p}^{\mathrm{CD}}(\mathrm{RHIC})=0.170,0.180,0.168 \mathrm{mb}$ and $\sigma_{p p}^{\mathrm{CD}}(\mathrm{LHC})=0.80,0.85,0.83 \mathrm{mb}$ for $f_{0}(1500), f_{0}(1710)$, and $X(1835)$, respectively.

In the next section, we compare the two production channels and investigate the main theoretical uncertainties. We provide estimates of cross sections and event rates for both processes for RHIC and LHC energies at the heavy-ion mode.

\section{RESULTS AND DISCUSSION}

In what follows, the numerical results for the two-photon and Pomeron-Pomeron processes are presented and discussed. In Table I, the cross sections for glueball production in photonphoton fusion at RHIC and LHC energies are shown. For RHIC we have considered the nominal center-of-mass energy of $200 \mathrm{GeV}$ for gold-gold collisions, and for LHC we take the planned nominal energy of $5500 \mathrm{GeV}$ in lead-lead collisions. The first value corresponds to the cross section obtained using a nonfactorizable photon flux (Cahn-Jackson) [28], and the second one refers to the factorizable flux as shown in Eqs. (1) and (2). The deviation is sizable for RHIC and LHC. The cross sections are sufficiently large for experimental measurement. The event rates can be obtained using the beam luminosity [9]: For LHC, one has $\mathcal{L}_{\mathrm{PbPb}}=5 \cdot 10^{26} \mathrm{~cm}^{-2} \mathrm{~s}^{-1}$, which produces the following number of events. One has $3.6 \times 10^{2}, 2 \times 10^{3}$, and 4 for $f_{0}(1500), f_{0}(1710)$, and $X(1835)$, respectively, in the nominal LHC running time with ions of $10^{6} \mathrm{~s}$ (one month). The event rates can be enhanced in a $p \mathrm{~Pb}$ mode, where the nominal beam luminosity is increased three orders of magnitude compared to the $\mathrm{PbPb}$ mode. The present calculation can be compared to previous studies on glueball production in heavy-ion collisions [29,30]. In general, the numerical results are similar to those computations, and the main deviation comes from the distinct estimates for the two-photon decays widths. A direct comparison can be done for the $f_{0}(1710)$ case, where in Ref. [30] one gets $48 \mathrm{nb}$ for RHIC and $2.3 \mu \mathrm{b}$ for LHC (using cut in impact parameter $b>2 R_{A}$ and using $\left.\Gamma_{\gamma \gamma} \simeq 4 \mathrm{eV}[30]\right)$.

For the convenience of phenomenologists, we provide here a parametrization of the ultraperipheral $A A$ cross section as a function of the resonance mass at the LHC energy. This makes the computation of event rates simple when the specific meson state and its two-photon decay width are provided. Using the Cahn-Jackson photon flux, we obtain in the interval $400 \leqslant M_{R} \leqslant 4000 \mathrm{MeV}$ the parametrization

$$
\frac{\sigma_{\mathrm{upc}}\left(A A \rightarrow R_{J}+A A\right)}{(2 J+1) \Gamma\left(R_{J} \rightarrow \gamma \gamma\right)}=\frac{\sigma_{0} M_{R}^{\beta}}{1+\left(M_{R} / 4\right)},
$$

where $\sigma_{0}=4.9147 \mathrm{mb} / \mathrm{GeV}$ and $\beta=-3.45335 ; \Gamma_{\gamma \gamma}$ and $M_{R}$ are the decay width and the resonance mass in units of giga-electron-volts, respectively. Several authors have argued for a low-lying scalar glueball, with mass between 500 and $1200 \mathrm{MeV}$ [1,2], depending on the components. The previous parametrization allows us to obtain estimates starting from a modeling for the two-photon width.

In Table II, the results for Pomeron-Pomeron production of glueball are presented. The estimates are shown for the inclusive (inc.) and exclusive (exc.) DPE as discussed in previous section. Namely, for the inclusive production, the Sudakov survival factor is not included (glueball is produced in association with Pomeron remnants), whereas for the exclusive case it is taken into account. In order to estimate the model dependence in the $\mathrm{CD}$ cross section, we have changed the soft Pomeron parameters in order to be consistent with the semi-hard Pomeron values considered in the DESY-HERA fits to diffractive deep inelastic scattering (DDIS). For instance, taking fit $\mathrm{A}$ of the $\mathrm{H} 1 \mathrm{C}$ Collaboration [31] parametrization for 
the diffractive structure function $F_{2}^{D(3)}$ one has $\epsilon=0.118$, $\alpha^{\prime}=0.06$, and $b=2.75 \mathrm{GeV}^{-2}$. Such a change enhances the cross section by a factor of 3 for $\mathrm{PbPb}$ collisions at the LHC. In Table II, the cross sections are presented, taking into account such a theoretical error band. The lower bound corresponds to soft Pomeron parameters, and the upper bound stands for the semihard Pomeron ones. For RHIC energy, the Pomeron-Pomeron contribution seems to be bigger than the photon-photon channel to a large extent. On the other hand, at the LHC they are competitive. However, the Pomeron contribution can be easily separated from photon channel by imposing a cut on the impact parameter of collision. After imposing this kinematic cut $\left(b>2 R_{A}\right)$, the Pomeron contribution is reduced as they are dominated by small impact parameter contributions.

The present result is difficult to compare directly to previous studies on Refs. [29,30]. Those authors did not include the survival probability gap in their calculations, and the theoretical approaches for Pomeron-Pomeron interaction are distinct. For instance, in Ref. [29], the IP IP $\rightarrow G$ cross section is obtained using the Pomeron-quark coupling like an isoscalar photon, which allows us to obtain the DPE cross section from the two-photon one. On the other hand, in Ref. [30], only the inclusive double Pomeron production is considered. Following that study, we can perform a closer comparison. The cross sections are computed there with inelastic scattering effects using the Glauber approximation (in Table III of Ref. [30]; see $\sigma_{A A}$ elastic), which is similar to the procedure presented here. After including a gap survival probability factor, one gets for the $f_{0}(1710)$ meson the values $1.23(3.04) \mathrm{mb}$ for RHIC (LHC), which is not so far from our results presented for inclusive production in Table II.

Finally, it is important to discuss the uncertainties in the current calculations and the experimental feasibility of detecting glueball candidates. The main uncertainty here is the model dependence on obtaining the two-photon and the two-gluon widths for a pure glueball meson. For the two-photon width, we considered the nonrelativistic gluon bound-state model of
Ref. [13], which could be a debatable issue and is far from being optimal. There are more modern approaches as reviewed in Ref. [2], but this is out of the scope of the present work. For the two-gluon widths, we obtained them from the quarkonium width based on a nonrelativistic bound-state calculation [22]. This type of matrix elements has been discussed in Ref. [32], giving rise to an effect of chiral suppression. We did not discuss the implication of those findings in the present calculation. Concerning the experimental detection, the advantage of the exclusive processes discussed here is clear: glueballs are probably being produced with a high cross section in inelastic collisions (in $p p$ or $A A$ reactions), but when the multiplicity is high the combinatorial background is overwhelming. In exclusive production, there is no combinatorial background. In the ultraperipheral two-photon production of glueballs, the final-state configuration is clear: nuclei remain intact after collision and a double large rapidity gap between them is present (glueball is centrally produced with a low $p_{T}$ transverse momenta spectrum). This type of measurement is already done at RHIC for photoproduction of vector mesons and exclusive dilepton production with a signal identification that is well understood [33]. The situation for DPE glueball production is similar, with the $p_{T}$ spectrum being broader than the processes initiated by two photons. Thus, a transverse momentum cut (and also impact parameter of collision) could separate the two channels (for a review of these issues, we refer readers to Ref. [34]).

\section{ACKNOWLEDGMENTS}

The authors thank Curtis A. Meyer, Nikolai Kochelev, Pedro Bicudo, and Dimiter Hadjimichef for comments and suggestions. M.V.T.M. acknowledges the Aristotle University of Thessaloniki and the organizers of the Low- $x$ Meeting (Kavala, Greece. June 21-27 2010) for their invitation, where this work was accomplished. This research was supported by CNPq, Brazil.
[1] V. Mathieu, N. Kochelev, and V. Vento, Int. J. Mod. Phys. E 18, 1 (2009).

[2] V. Crede and C. A. Meyer, Prog. Part. Nucl. Phys. 63, 74 (2009).

[3] R. Barate et al. (ALEPH Collaboration), Phys. Lett. B 472, 189 (2000).

[4] F. E. Close and A. Kirk, Phys. Lett. B 483, 345 (2000).

[5] C. Amsler and F. E. Close, Phys. Lett. B 353, 385 (1995).

[6] M. Ablikim et al. (BES Collaboration), Phys. Rev. Lett. 95, 262001 (2006).

[7] N. Kochelev and D. P. Min, Phys. Lett. B 633, 283 (2006).

[8] B. A. Li, Phys. Rev. D 74, 034019 (2006).

[9] G. Baur, K. Hencken, D. Trautmann, S. Sadovsky, Y. Kharlov, Phys. Rep. 364, 359 (2002); C. A. Bertulani, S. R. Klein, and J. Nystrand, Annu. Rev. Nucl. Part. Sci. 55, 271 (2005); K. Hancken et al., Phys. Rep. 458, 1 (2008).

[10] S. J. Brodsky, T. Kinoshita, and H. Terazawa, Phys. Rev. Lett. 25, 972 (1970); Phys. Rev. D 4, 1532 (1971).
[11] K. Nakamura et al. (Particle Data Group), J. Phys. G: Nucl. Part. Phys. 37, 075021 (2010).

[12] M. B. Çakir and G. R. Farrar, Phys. Rev. D 50, 3268 (1994).

[13] E. H. Kada, P. Kessler, and J. Parisi, Phys. Rev. D 39, 2657 (1989).

[14] C. A. Bertulani, Phys. Rev. C 79, 047901 (2009).

[15] P. V. Landshoff and O. Nachtmann, Z. Phys. C 35, 405 (1987).

[16] A. Bialas and P. V. Landshoff, Phys. Lett. B 256, 540 (1991).

[17] A. Szczurek and P. Lebiedowicz, Nucl. Phys. A 826, 101 (2009).

[18] E. Gotsman, E. Levin, and U. Maor, Phys. Lett. B 438, 229 (1998).

[19] A. B. Kaidalov, V. A. Khoze, A. D. Martin, and M. G. Ryskin, Eur. Phys. J. C 21, 521 (2001).

[20] E. Gotsman, E. Levin, U. Maor, and A. Prygarin, arXiv:hep-ph/0511060.

[21] V. A. Khoze, A. D. Martin, M. G. Ryskin, and W. J. Stirling, Eur. Phys. J. C 35, 211 (2004). 
[22] F. E. Close, G. R. Farrar, and Z. Li, Phys. Rev. D 55, 5749 (1997).

[23] A. Bzdak, Phys. Lett. B 615, 240 (2005).

[24] V. A. Khoze, A. D. Martin, and M. G. Ryskin, Eur. Phys. J. C 23, 311 (2002).

[25] C. Pajares and V. A. Ramallo, Phys. Lett. B 107, 238 (1981);

C. Pajares and A. V. Ramallo, Phys. Rev. D 31, 2800 (1985);

M. A. Braun and C. Pajares, Nucl. Phys. A 532, 678 (1991).

[26] S. Y. Shmakov et al., Comput. Phys. Commun. 54, 125 (1989).

[27] R. Engel, Z. Phys. C 66, 203 (1995).
[28] R. N. Cahn and J. D. Jackson, Phys. Rev. D 42, 3690 (1990). [29] A. A. Natale, Phys. Lett. B 362, 177 (1995).

[30] A. J. Schramm, J. Phys. G: Nucl. Part. Phys. 25, 1965 (1999).

[31] A. Aktas et al. (H1 Collaboration), Eur. Phys. J. C 48, 715 (2006).

[32] M. S. Chanowitz, Phys. Rev. Lett. 95, 172001 (2005); Int. J. Mod. Phys. A 21, 5535 (2006).

[33] J. Nystrand, Nucl. Phys. A 787, 29 (2007).

[34] M. G. Albrow, T. D. Coughlin, and J. R. Forshaw, Prog. Part. Nucl. Phys. 65, 149 (2010). 